\title{
Phytoprotection
}

\section{Comparaison de plans d'échantillonnage séquentiel binomial et de type Iwao pour le dépistage du thrips de l'oignon (Thrips tabaci) [Thysanoptera : Thripidae] sur l'oignon}

\section{F. Fournier, G. Boivin et R.K. Stewart}

Volume 75, numéro 2, 1994

URI : https://id.erudit.org/iderudit/706053ar

DOI : https://doi.org/10.7202/706053ar

Aller au sommaire du numéro

Éditeur(s)

Société de protection des plantes du Québec (SPPQ)l

ISSN

0031-9511 (imprimé)

1710-1603 (numérique)

Découvrir la revue

Citer cet article

Fournier, F., Boivin, G. \& Stewart, R. (1994). Comparaison de plans d'échantillonnage séquentiel binomial et de type Iwao pour le dépistage du thrips de l'oignon (Thrips tabaci) [Thysanoptera : Thripidae] sur l'oignon. Phytoprotection, 75(2), 69-78. https://doi.org/10.7202/706053ar
Résumé de l'article

Des différences transitoires de densité du thrips de l'oignon (Thrips tabaci) ont été observées entre les bordures et le centre de certains champs d'oignon (Allium cepa). Les populations de thrips sont constituées d'agrégats dispersés de façon contagieuse à l'intérieur des champs d'oignon. Deux types de plans d'échantillonnage séquentiel ont été établis pour le $T$. tabaci sur l'oignon: plan binomial basé sur la présence d'au moins 5 thrips plant d'oignon ${ }^{-1}$ et plan de type Iwao nécessitant le décompte de tous les thrips présents. Les limites d'acceptation de ces plans ont été calculées pour des seuils économiques de 0,9 et 2,2 thrips feuille ${ }^{-1}$ Ces plans ont été validés dans des champs abritant des populations entre 0,01 et 32,33 thripsfeuille ${ }^{-1}$. Les plans de type binomial se sont avérés aussi fiables que les plans séquentiels avec des taux d'erreur ne dépassant jamais $4 \%$, ce qui est inférieur aux niveaux théoriques choisis de 5 et $10 \%$. De bonnes prises de décision ont été obtenues dans 90,4 et $83,6 \%$ des cas à l'aide des plans par décompte, et dans 84,8 et $82,4 \%$ des cas à l'aide des plans binomiaux pour les seuils de 0,9 et 2,2 thrips feuille $^{-1}$, respectivement. Le nombre moyen d'échantillons à prélever pour prendre une décision oscille entre 10 et 14 plants d'oignon selon le plan employé. L'emploi des plans binomiaux peut entraîner des réductions de l'effort de comptage des thrips pouvant atteindre jusqu'à 62 et $88 \%$ pour les seuils de 0,9 et 2,2 thrips feuille ${ }^{-1}$, respectivement. 


\title{
Comparaison de plans d'échantillonnage séquentiel binomial et de type Iwao pour le dépistage du thrips de l'oignon (Thrips tabaci) [Thysanoptera: Thripidae] sur l'oignon
}

\author{
François Fournier ${ }^{1}$, Guy Boivin² et Robin K. Stewart ${ }^{3}$
}

Reçu 1993-10-13; accepté 1994-11-01

Des différences transitoires de densité du thrips de l'oignon (Thrips tabaci) ont été observées entre les bordures et le centre de certains champs d'oignon (Allium cepa). Les populations de thrips sont constituées d'agrégats dispersés de façon contagieuse à l'intérieur des champs d'oignon. Deux types de plans d'échantillonnage séquentiel ont été établis pour le T. tabaci sur l'oignon: plan binomial basé sur la présence d'au moins 5 thrips plant d'oignon ${ }^{-1}$ et plan de type Iwao nécessitant le décompte de tous les thrips présents. Les limites d'acceptation de ces plans ont été calculées pour des seuils économiques de 0,9 et 2,2 thrips feuille-1. Ces plans ont été validés dans des champs abritant des populations entre 0,01 et 32,33 thrips feuille $^{-1}$. Les plans de type binomial se sont avérés aussi fiables que les plans séquentiels avec des taux d'erreur ne dépassant jamais $4 \%$, ce qui est inférieur aux niveaux théoriques choisis de 5 et $10 \%$. De bonnes prises de décision ont été obtenues dans 90,4 et $83,6 \%$ des cas à l'aide des plans par décompte, et dans 84,8 et $82,4 \%$ des cas à l'aide des plans binomiaux pour les seuils de 0,9 et 2,2 thrips feuille ${ }^{-1}$, respectivement. Le nombre moyen d'échantillons à prélever pour prendre une décision oscille entre 10 et 14 plants d'oignon selon le plan employé. L'emploi des plans binomiaux peut entraîner des réductions de l'effort de comptage des thrips pouvant atteindre jusqu'à 62 et $88 \%$ pour les seuils de 0,9 et 2,2 thrips feuille-1, respectivement.

Fournier, F., G. Boivin, and R.K. Stewart. 1994. Comparison of binomial and Iwao type sequential sampling plans for monitoring onion thrips (Thrips tabaci) [Thysanoptera: Thripidae] in onions. PHYTOPROTECTION 75: 69-78.

Transient differences in onion thrips (Thrips tabaci) densities were observed between the margins and the centre of some onion (Allium cepa) fields. Onion thrips populations consist in aggregates contagiously dispersed within onion fields. Two types of sequential sampling plans were established for T. tabaci in onions: a binomial plan based on the presence of

1. Département des sciences en ressources naturelles (entomologie), Campus Macdonald de I'Université McGill, 21 111, chemin Lakeshore, Sainte-Anne-de-Bellevue (Québec), Canada H9X 3V9. Adresse actuelle: Services Bio-Contrôle inc., 430, boul. Gouin, Saint-Jean-surRichelieu (Québec), Canada J3B 3E6

2. Agriculture et Agro-alimentaire Canada, 430, boul. Gouin, Saint-Jean-sur-Richelieu (Québec), Canada J3B 3E6

3. Département des sciences en ressources naturelles (entomologie), Campus Macdonald de I'Université McGill, 21 111, chemin Lakeshore, Sainte-Anne-de-Bellevue (Québec), Canada H9X 3V9 
5 thrips plant $^{-1}$, and one of the Iwao type, requiring counts of all thrips present. Acceptation boundaries for these plans were calculated for economic thresholds of 0.9 and 2.2 thrips leaf $^{-1}$. Plans were validated in fields with thrips populations varying between 0.01 and 32.33 thrips leaf $^{-1}$. Binomial sequential sampling plans were found to be as reliable as plans requiring counts of all thrips, with an error rate not exceeding $4 \%$, a level below the theoretical error rates of 5 and $10 \%$ used in the calculations. Correct decisions were reached in 90.4 and $83.6 \%$ of the situations with plans requiring counts of all thrips, and in 84.8 and $82.4 \%$ of the situations with binomial plans for economic thresholds of 0.9 and 2.2 thrips leaf $^{-1}$, respectively. The average number of samples needed to reach a decision varied between 10 and 14 plants according to the plan used. Use of binomial plans can save up to 62 and $88 \%$ of the counting effort for thresholds of 0.9 and 2.2 thrips leaf $^{-1}$, respectively.

\section{INTRODUCTION}

Le thrips de l'oignon (Thrips tabaci Lindeman) [Thysanoptera: Thripidae] est, après la mouche de l'oignon (Delia antiqua Meigen) [Diptera: Anthomyiidael, l'insecte ravageur le plus important de I'oignon (Allium cepa L.) au Québec (Boivin et Brodeur 1992). Les thrips s'attaquent au feuillage de l'oignon et ont un impact indirect sur les rendements. Des pertes de rendement de 28 à $40 \%$ ont été enregistrées au Texas avec des moyennes saisonnières dépassant 24 thrips plant $^{-1}$ (Edelson et al. 1986, 1989). Au Québec, ces pertes ont été de $43 \%$ lors d'une importante infestation de thrips atteignant une moyenne saisonnière de plus de 150 thrips plant ${ }^{-1}$ (Fournier 1993). En plus de l'abondance des thrips, d'autres facteurs peuvent agir pour déterminer l'impact des thrips sur les rendements, comme le stade phénologique de l'oignon au moment de l'infestation (Kendall et Capinera 1987) ainsi que les conditions climatiques (Edelson et al. 1989).

Shelton et al. (1987) ont fixé empiriquement à 3 thrips feuille ${ }^{-1}$ le seuil d'action dynamique contre le $T$. tabaci dans l'État de New York. Des seuils économiques ont été calculés à la suite d'importantes infestations du T. tabaci durant deux étés chauds et secs au Québec. Ce seuil a été établi à 0,9 thrips feuille ${ }^{-1}$ durant un été marqué par une sécheresse sévère ( 3 sem sans précipitations) au stade de 6 à 8 feuilles vivantes de
I'oignon, et à 2,2 thrips feuille-1 durant un été à pluviométrie déficitaire mais régulière (Fournier 1993).

Deux plans d'échantillonnage séquentiel qui nécessitent le décompte des thrips ont été proposés pour le thrips de I'oignon sur l'oignon. À partir des paramètres d'une dispersion contagieuse des thrips établis par Suman et al. (1980) dans des parcelles avec une densité maximale de 7 thrips plant ${ }^{-1}$, Suman et Wahi (1981) ont calculé les limites d'acceptation de leur plan. L'emploi de ce plan avec un seuil supérieur à 7 thrips plant ${ }^{-1}$ suppose que la dispersion spatiale du thrips est la même à de plus hautes densités et peut entraîner des niveaux d'erreur supérieurs à ceux prévus par le plan d'échantillonnage (Bates et al. 1991). Shelton et al. (1987) ont construit leur plan sur la base d'une dispersion aléatoire des thrips établie par le décompte absolu des thrips et une unité d'échantillonnage de trois plants d'oignon. Idéalement, la même méthode d'échantillonnage devrait être utilisée pour l'établissement du seuil économique, pour la description de la dispersion spatiale et pour la mise en application du plan d'échantillonnage (Boivin et Vincent 1983). Pour des raisons de simplicité et de rapidité d'exécution, nous avons utilisé le décompte visuel des thrips avec un plant d'oignon comme unité d'échantillonnage pour établir les seuils économiques puisqu'il existe une relation linéaire entre les décomptes visuel et absolu des thrips sur I'oignon (Edelson 1985). 
Avec des insectes aussi petits que les thrips et un seuil économique assez élevé, le décompte des thrips peut cependant prendre beaucoup de temps lorsque les populations approchent du seuil économique. Des programmes d'échantillonnage séquentiel binomiaux basés sur la présence ou l'absence de ravageurs ont été développés pour des espèces petites et abondantes comme les acariens (Belchinski et Stoltz 1985) et les pucerons (Wilson et al. 1983). L'élaboration de tels plans requiert l'existence d'une relation mathématique entre la densité du ravageur et la proportion de plants infestés qui tienne aussi compte de la dispersion spatiale du ravageur.

Nous avons étudié la dispersion spatiale des thrips de l'oignon dans des champs d'oignon commerciaux. L'information ainsi recueillie a servi au développement de deux plans d'échantillonnage séquentiel: un plan basé sur le décompte visuel des thrips et un plan binomial. Ces plans sont comparés sur la base de leur efficacité et de leur rapidité d'exécution pour les seuils économiques de 0,9 et 2,2 thrips feuille ${ }^{-1}$.

\section{MATÉRIEL ET MÉTHODES}

Dix champs d'oignon commerciaux du sud de Montréal ont été visités une ou deux fois par semaine entre la mi-juin et la mi-août pour un total de 64 visites en 1988 (six champs) et de 37 visites en 1989 (quatre champs). Cette période couvre le développement de l'oignon, du stade de la $4^{\mathrm{e}}$ ou $5^{\mathrm{e}}$ feuille à la pleine maturité des bulbes. La dimension des champs variait entre 1,5 et 7,5 ha. Différents cultivars d'oignon jaune ont été utilisés et les pratiques agricoles courantes dans cette culture au Québec ont été effectuées (C.P.V.Q. 1987).

Lors de la première visite, une bordure de $5 \mathrm{~m}$ a été délimitée sur chaque côté du champ, divisant ainsi le champ en cinq secteurs: quatre bordures et le centre. Cette procédure visait à vérifier $s^{\prime} i l$ n'existait pas une plus grande densité de thrips dans les bordures, spécialement en début d'infestation. Lors de chaque visite, 50 plants ont été choisis au hasard sur toute l'étendue du champ. Par conséquent, il n'y avait pas nécessairement un nombre égal d'échantillons par secteur. Pour chaque plant, le stade phénologique (nombre de feuilles vivantes et mortes), la position dans le champ (bordure ou centre), de même que le décompte visuel des thrips (larves et adultes confondus) étaient notés.

\section{Distribution spatiale}

L'uniformité de la densité de $T$. tabaci entre les différents secteurs de chaque champ a été vérifiée, pour chaque date d'échantillonnage, par une analyse de variance à un critère de classification de Kruskall-Wallis appliquée aux rangs des densités de thrips. Les égalités de rangs ont nécessité un ajustement du $\mathrm{H}$ calculé (Daniel 1978). Les différences de densité ont été établies à I'aide du test proposé par Dunn (1964) en faisant, ici aussi, les ajustements requis par l'égalité des rangs.

\section{Échantillonnage séquentiel par décompte}

Iwao (1968) a démontré pour plusieurs organismes l'existence d'une relation linéaire entre la densité moyenne $(\bar{x})$ et I'encombrement moyen ( $(*)$ ). L'ordonnée à l'origine, appelée indice de contagion, caractérise l'unité de base de la population à une densité infinitésimale, et la pente, appelée coefficient de dispersion, décrit la dispersion de ces unités de base dans l'espace. Ces paramètres sont intégrés dans les formules fournies par Iwao (1975) pour calculer les limites d'acceptation du plan d'échantillonnage et fixer le nombre maximum d'échantillons à inspecter dans l'éventualité d'un niveau d'infestation près du seuil économique.

\section{Échantillonnage séquentiel binomial}

L'échantillonnage binomial repose sur la simple présence ou absence de ravageurs. Pour être fiable, une relation doit exister entre la densité du ravageur et la proportion de plantes infestées. Cette relation a été estimée à l'aide de l'Éq. [1], telle que proposée par Wilson et Room (1983):

$$
P=1-e\left(-\bar{x} \frac{\ln \left(a \bar{x}^{b-1}\right)}{a \bar{x}^{b-1}-1}\right)
$$


où $\mathrm{P}$ est la proportion de plants infestés à une densité moyenne $\bar{x}$ de thrips plant $^{-1}$ dans un champ donné. Les paramètres $a$ et $b$ de l'Éq. [1] peuvent être estimés par l'équation de Taylor (1961), b étant un indice de dispersion, ou par itération (Wilson et al. 1983).

Les limites d'acceptation du plan séquentiel binomial sont calculées à l'aide de l'Éq. [2], en construisant un intervalle de confiance pour la proportion de plants infestés correspondant au seuil économique $\left(P_{s}\right)$ multipliée par $n$, le nombre de plants inspectés (Belchinski et Stoltz 1985):

$$
\begin{aligned}
& \text { Limites d'acceptation }= \\
& \mathrm{P}_{\mathrm{s}} \mathrm{n} \pm t_{(\alpha, \infty)} \mathrm{n}\left[\left[\left[\mathrm{P}_{\mathrm{s}}\left(1-\mathrm{P}_{\mathrm{s}}\right)\right] / \mathrm{n}\right]^{1 / 2}\right]
\end{aligned}
$$

Le nombre maximum $\left(N_{\max }\right)$ de plants à inspecter pour un plan binomial est calculé selon l'Éq. [3] (Karandinos 1976):

$$
N_{\max }=\left[t^{2}(\alpha, \propto) P_{s}\left(1-P_{s}\right)\right] / h^{2}
$$

où $h$ est la précision désirée sur l'estimation de la proportion lorsque celleci est égale au seuil économique.

\section{Validation des plans d'échantillonnage}

Afin de valider les plans d'échantillonnage, 18 autres champs d'oignon commerciaux ont été visités à une ou deux reprises pour un total de 25 visites en 1989. Une estimation de la population de $T$. tabaci a été effectuée par le décompte visuel des thrips sur 100 plants d'oignon choisis au hasard. La validation de chaque combinaison plan d'échantillonnage séquentiel - seuil économique a été effectuée par 250 simulations. Pour chaque visite, des plantes ont été choisies au hasard de façon séquentielle parmi l'échantillon de 100 plantes. Le décompte de thrips ainsi obtenu a été comparé avec les limites du plan à valider jusqu'à ce qu'une décision soit prise ou à l'atteinte du $\mathrm{N}_{\max }$. Cette procédure a été répétée 10 fois pour chaque visite. Les décisions prises sur la base des plans d'échantillonnage séquentiel ont été comparées avec la décision prise sur la base de la moyenne des 100 plants. Le nombre minimum d'échantillons à inspecter avant toute prise de décisions a été fixé à 10 , ce qui résulte généralement en des risques d'erreur inférieurs au niveau théorique (Fowler et Lynch 1987).

\section{RÉSULTATS ET DISCUSS\|ON}

\section{Distribution spatiale}

Aucune différence de densité des thrips n'a été détectée entre les différents secteurs des champs d'oignon pour 43 des 58 dates d'échantillonnage avec thrips en 1988, ni pour 24 des 29 dates d'échantillonnage pour lesquelles une telle comparaison était possible en 1989 (tableaux 1, 2). Des différences de densité de population ont été détectées dans tous les champs en 1988. Cela s'est produit à une seule occasion dans les champs 3 et 4, et à deux dates d'échantillonnage espacées de plus de 1 mo dans le champ 5. Dans les autres champs, 1,2 et 6 , des différences de densité ont été détectées pendant au moins deux échantillonnages consécutifs. En 1989, la densité des thrips a été uniforme tout au long de la saison entre les différents secteurs des champs 2 et 3. Des différences de densité sont apparues au mois de juillet dans les champs 1 et 4 pour se maintenir au moins 2 sem. Toutes les différences de densité détectées entre les différents secteurs ont impliqué une ou deux bordures de champ ayant une densité de thrips supérieure à une autre bordure ou au centre du champ d'oignon. Prises individuellement, ces différences $n$ 'ont pas persisté pendant plus de deux échantillonnages consécutifs.

Des densités inégales de thrips entre les différents secteurs d'un champ d'oignon peuvent être attribuées à la biologie de l'insecte, à des facteurs externes comme le vent, ou aux deux à la fois. Shelton et al. (1987) ont aussi rapporté un effet de bordure semblable dans certains champs d'oignon de l'État de New York. La migration du T. tabaci 
Tableau 1. Valeur de la variable $H$ du test de Kruskall-Wallis (ajustée en fonction de l'égalité des rangs) pour la comparaison de la densité des thrips entre les différents secteurs de champs d'oignon du sud de Montréal (Québec) en 1988

\begin{tabular}{|c|c|c|c|c|c|c|}
\hline \multirow[b]{2}{*}{ Semaine } & \multicolumn{6}{|c|}{ Champ } \\
\hline & 1 & 2 & 3 & 4 & 5 & 6 \\
\hline $06-12$ & $0^{\mathrm{a}}$ & & 0 & & 0 & \\
\hline 06-19 & 4,00 & 0 & 0 & 3,54 & 6,14 & 2,89 \\
\hline $06-26$ & 2,59 & 1,23 & 3,91 & 1,17 & 4,93 & 3,05 \\
\hline $07-03$ & 4,19 & 4,05 & 4,25 & 2,66 & $12,71^{*}$ & 2,07 \\
\hline $07-10$ & 7,72 & $\begin{array}{l}4,84 \\
1,49\end{array}$ & 1,85 & $\begin{array}{c}14,97 * * \\
2,84\end{array}$ & 4,82 & $\begin{array}{l}2,10 \\
5,96\end{array}$ \\
\hline $07-17$ & $11,12^{*}$ & 2,76 & 3,15 & 4,28 & $\begin{array}{l}6,16 \\
4,95\end{array}$ & $\begin{array}{l}1,93 \\
3,58\end{array}$ \\
\hline $07-24$ & $10,43^{*}$ & $10,11^{*}$ & 1,82 & 4,51 & $\begin{array}{l}4,51 \\
0,55\end{array}$ & $\begin{array}{c}4,13 \\
11,16^{*}\end{array}$ \\
\hline $07-31$ & $12,32^{*}$ & $15,30 * *$ & $9,57^{*}$ & 2,59 & $\begin{array}{l}4,87 \\
4,21\end{array}$ & $\begin{array}{c}10,40^{*} \\
2,87\end{array}$ \\
\hline 08-07 & $9,33^{*}$ & $10,62^{*}$ & 0,24 & 8,15 & $\begin{array}{l}3,13 \\
9,09 *\end{array}$ & $\begin{array}{c}4,79 \\
10,23^{*}\end{array}$ \\
\hline $08-21$ & & $11,10^{*}$ & & & & \\
\hline
\end{tabular}

Population de thrips nulle.

${ }^{*},{ }^{*}$ Différence significative aux niveaux $\alpha=0,05$ et $\alpha=0,01$, respectivement.

Tableau 2. Valeur de la variable $\mathbf{H}$ du test de Kruskall-Wallis (ajustée en fonction de l'égalité des rangs) pour la comparaison de la densité des thrips entre les différents secteurs de champs d'oignon du sud de Montréal (Québec) en 1989

\begin{tabular}{|c|c|c|c|c|}
\hline \multirow[b]{2}{*}{ Semaine } & \multicolumn{4}{|c|}{ Champ } \\
\hline & 1 & 2 & 3 & 4 \\
\hline $06-25$ & $\begin{array}{l}1,05 \\
-{ }^{a}\end{array}$ & $\begin{array}{c}0,52 \\
-\end{array}$ & 1,06 & 6,05 \\
\hline $07-02$ & - & - & 6,85 & \\
\hline $07-09$ & - & 2,88 & 0,87 & 2,13 \\
\hline $07-16$ & $\begin{array}{l}1,09 \\
3,46\end{array}$ & 2,68 & 6,65 & $15,49^{* *}$ \\
\hline $07-23$ & $\begin{array}{l}3,99 \\
6,64^{*}\end{array}$ & $\begin{array}{l}2,61 \\
6,98\end{array}$ & 8,27 & $22,10 * *$ \\
\hline $07-30$ & & & 7,03 & $13,84^{* *}$ \\
\hline $08-06$ & $10,62 * *$ & & - & 3,52 \\
\hline $08-13$ & 0,10 & 5,33 & $\begin{array}{l}4,13 \\
3,13\end{array}$ & \\
\hline $08-20$ & & 2,93 & 3,28 & \\
\hline
\end{tabular}

Échantillonnage du centre du champ seulement empêchant toute comparaison entre les secteurs.

*, * Différence significative aux niveaux $\alpha=0,05$ et $\alpha=0,01$, respectivement. 
vers des champs d'oignon a été rapportée à l'atteinte de la maturité de champs avoisinants de céréales, de foin ou de luzerne, ou à la suite de leur fauchage (Boyce et Miller 1953; North et Shelton 1986). La migration et le vol passif des thrips, qui sont parmi les moins bons voiliers des insectes (Lewis 1973), pourraient résulter en une colonisation inégale de certains champs d'oignon, les bordures exposées au vent accueillant un plus grand nombre d'individus. Nos données indiquent qu'il existe une grande variabilité sur ce point entre les différents champs.

\section{Échantillonnage séquentiel par décompte}

Les populations de T. tabaciéchantillonnées ont varié entre 0 et 22,4 thrips plant $^{-1}$ en 1988, et entre 0 et 163,9 thrips plant $^{-1}$ en 1989. Cette vaste gamme de densités inclut les valeurs des seuils économiques pour lesquels les plans sont développés. Les champs qui ont servi à la validation des plans d'échantillonnage séquentiel abritaient des populations oscillant entre 0,4 et plus de 250 thrips plant $^{-1}$.

Les données de tous les champs ont été combinées pour calculer les paramètres de la régression linéaire de $\stackrel{*}{x}$ sur $\bar{x}$. Cette procédure fournit les paramètres de la dispersion spatiale de $T$. tabaci dans un champ d'oignon moyen couvrant une vaste gamme de conditions agronomiques. Une équation hautement significative a été établie $(* \vec{x}=$ $5,15+2,12 \bar{x} ; \mathrm{n}=95, r=0,98, P<0,01)$. $L$ 'indice de contagion statistiquement supérieur à $0(t=1,66 ; P<0,01)$ indique que l'unité de base des populations de thrips est constituée d'agrégats d'individus. Une dispersion contagieuse de ces agrégats est révélée par un coefficient de dispersion significativement supérieur à $1(t=23,10 ; P<0,01)$. Ce dernier résultat est en accord avec ceux d'Edelson et al. (1986) et de Suman et al. (1980) couvre une gamme de densités de population plus large. Shelton et al. (1987) observent aussi une dispersion contagieuse lorsqu'un seul plant d'oignon est utilisé au lieu de trois comme unité d'échantillonnage, cette dernière masquant la contagion des thrips.
La faible capacité de vol des adultes résulte sûrement en une dispersion limitée de ceux-ci. Lorsqu'une femelle atterrit sur un plant d'oignon, les plants rapprochés ont une probabilité plus élevée d'être colonisés par cet individu et sa progéniture que des plants éloignés. L'agrégation des thrips s'explique par leur haute fécondité et par la faible mobilité des stades immatures qui peuvent constituer jusqu'à $90 \%$ de la population de T. tabaci (Quartey 1982). Ces caractéristiques biologiques sont comparables à celles des pucerons pour lesquels des dispersions contagieuses d'agrégats d'individus ont aussi été rapportées (Maiteki et Lamb 1987; Wilson et al. 1983).

Avec un taux d'erreur de $10 \%$, pour des oignons de 6 à 10 feuilles vivantes, le nombre maximum de plants à inspecter varie entre 44 et 55 plants pour le seuil de 0,9 thrips feuille ${ }^{-1}$, et entre 51 et 58 plants pour le seuil de 2,2 thrips feuille ${ }^{-1}$. Ces deux plans ont été validés et trouvés fiables avec des taux d'erreur ne dépassant pas 2\% (tableau 3 ). Pour toutes les situations d'indécision, la densité des thrips était à l'intérieur de la précision choisie sur le seuil économique. Dans ces situations, le nombre d'individus à dénombrer sans pour autant prendre de décision peut atteindre 528 et 1427 thrips pour les seuils de 0,9 et 2,2 thrips feuille ${ }^{-1}$, respectivement.

\section{Échantillonnage séquentiel binomial}

Une relation significative a été établie par itération entre la densité de population de thrips et la proportion de plants infestés par un thrips ou plus (fig. 1). Pour estimer précisément une densité de population, la proportion de plants infestés correspondante ne doit pas excéder $80 \%$ (Southwood 1978). À cette proportion correspond une densité maximale de 6 thrips plant ${ }^{-1}$ lorsqu'un plant est considéré infesté par la présence d'un seul thrips. Cette densité est inférieure aux deux seuils économiques pour des plants de plus de six feuilles vivantes et limiterait les plans d'échantillonnage à des niveaux d'infestation non dommageables. La densité maximale détectable peut être accrue en augmentant le nombre minimum de 
thrips présents sur un plant pour le considérer infesté. Des plans binomiaux basés sur la présence d'au moins 5 acariens feuille ${ }^{-1}$ ont ainsi été développés par Hollingsworth et Berry (1982) et par Belchinski et Stoltz (1985). Avec 5 thrips plant ${ }^{-1}$ comme condition pour considérer un plant d'oignon infesté, il est possible d'estimer précisément des densités de thrips allant jusqu'à 29,2 thrips plant ${ }^{-1}$ (fig. 1). Cependant, cette condition rend inutilisable le plan binomial avec un seuil de 0,9 thrips feuille ${ }^{-1}$ pour des plants d'oignon de 5 feuilles vivantes ou moins, le nombre minimum de thrips excédant déjà le seuil économique. Le plan par décompte doit donc être utilisé pour ces situations. Sous les conditions climatiques du Québec, les infestation de thrips atteignent rarement le niveau de 5 thrips plant ${ }^{-1}$ avant le stade de la $6^{\mathrm{e}}$ feuille.
Avec le même taux d'erreur et la même précision sur les seuils économiques pour des oignons ayant entre 6 et 10 feuilles vivantes, le nombre maximum d'échantillons à inspecter varie de 9 à 12 plants et de 24 à 34 plants pour les seuils de 0,9 et 2,2 thrips feuille $^{-1}$, respectivement. Selon la méthode d'lwao, le calcul du $\mathrm{N}_{\max }$ incorpore les paramètres de la dispersion spatiale du ravageur, une dispersion de plus en plus contagieuse nécessitant un effort d'échantillonnage plus important pour une précision donnée. Dans l'échantillonnage binomial, les paramètres de la dispersion spatiale servent à établir la relation densité de population - proportion de plants infestés et le $\mathrm{N}_{\max }$ n'est fixé qu'en fonction de la précision désirée sur l'estimation de la proportion correspondant au seuil économique.

Tableau 3. Distribution des décisions prises après 250 simulations pour différents plans d'échantillonnage séquentiel du Thrips tabaci sur I'oignon

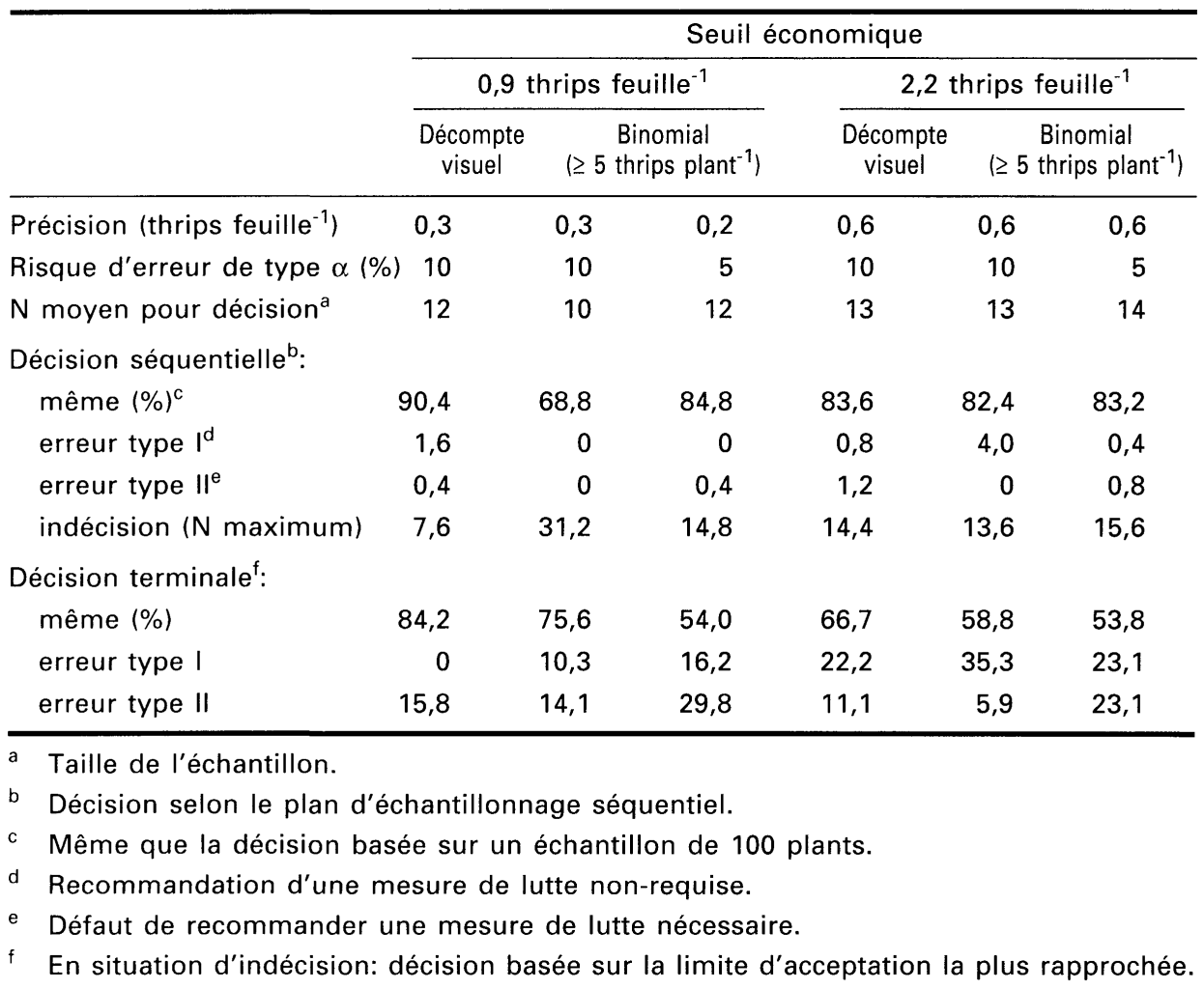




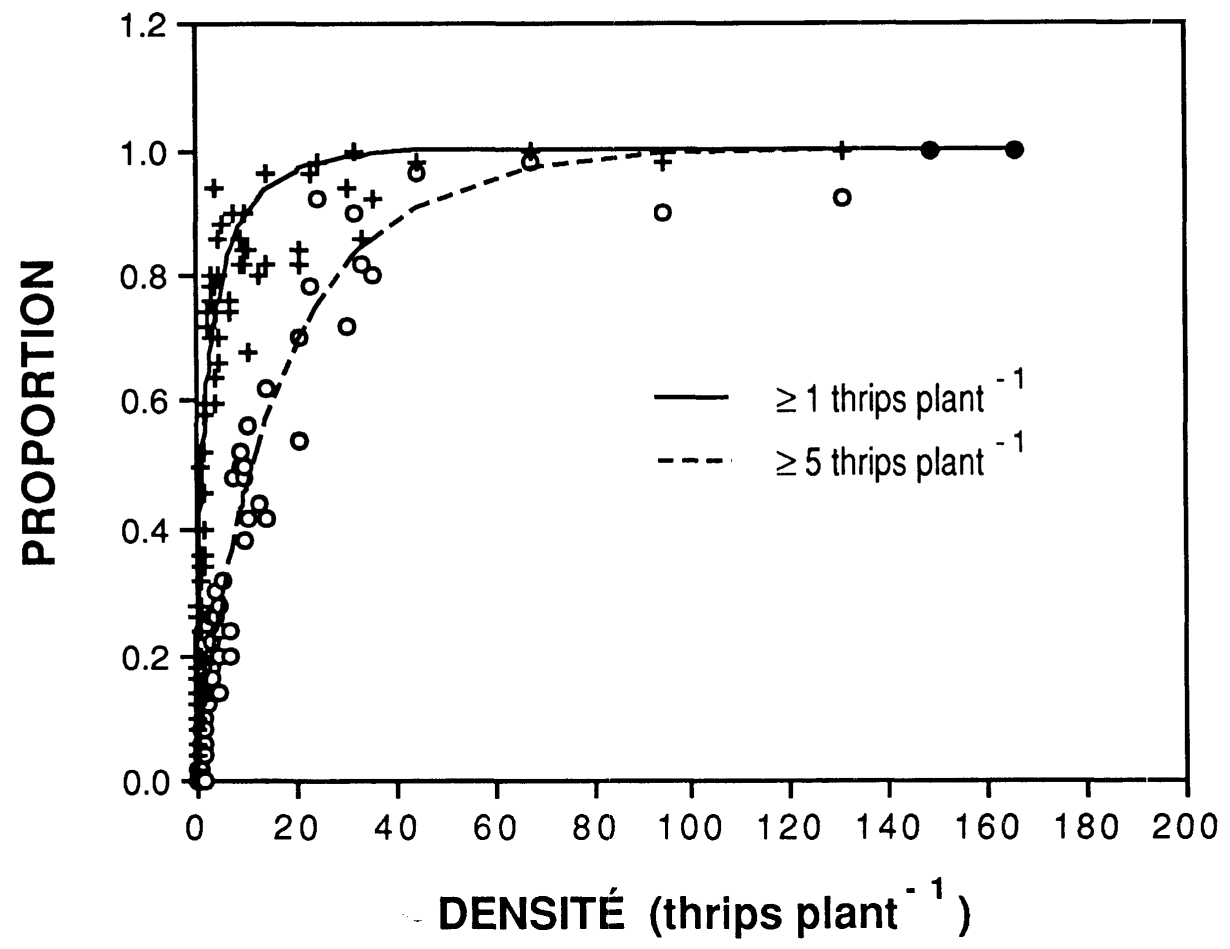

Figure 1. Relation entre la densité moyenne $(\bar{x})$ de $T$. tabaci par plant et la proportion (P) de plants infestés avec $\geq 1$ thrips plant $^{-1}\left(P=1-e\left[-\bar{x} \ln \left(3,15 \bar{x}^{1,60-1}\right) /\left(3,15 \bar{x}^{1,60-1}-1\right)\right] ; n=95\right.$; $r=0,99, P<0,01)$ et de plants infestés avec $\geq 5$ thrips plant ${ }^{-1}\left(P=1-\mathrm{e}\left[-\bar{x} \ln \left(55,01 \bar{x}^{1,11-1}\right) /(55,01\right.\right.$ $\left.\left.\overline{\mathrm{x}}^{1,11-1}-1\right)\right] ; n=95 ; r=0,99 ; P<0,01$ )

Après simulation, les plans binomiaux s'avèrent aussi fiables que ceux d'Iwao avec des taux d'erreur observés ne dépassant pas $4 \%$ (tableau 3 ). L'efficacité du plan binomial, mesurée en proportion de la prise de la bonne décision pour le seuil de 2,2 thrips feuille ${ }^{-1}$ est comparable à celle de son équivalent par décompte. Pour le seuil de 0,9 thrips feuille ${ }^{-1}$, le plan binomial entraîne une diminution de $21,6 \%$ dans la proportion de prise de la bonne décision. Dans ce cas, la haute proportion de situations d'indécision qui résulte du faible effort d'échantillonnage pourrait être réduite par l'augmentation du $\mathrm{N}_{\max }$. Ceci entraînerait aussi un meilleur taux d'erreur du plan binomial, ou une précision accrue sur la proportion seuil, ou les deux à la fois. Avec un taux d'erreur de $5 \%$ et une précision de 0,2 thrips feuille ${ }^{-1}$ sur le seuil, l'effort d'échantillonnage peut être accru entre 27 et 40 plants selon le stade des oignons. Après simulation, le risque d'erreur demeure sous le niveau théorique et la proportion de prise de la bonne décision augmente à $84,8 \%$, un niveau presque équivalent au plan par décompte (tableau 3). L'augmentation de l'effort d'échantillonnage (entre 35 et 48 plants) pour le seuil de 2,2 thrips feuille ${ }^{-1}$ permet de réduire le risque d'erreur théorique à $5 \%$ pour la même précision sur le seuil. Après simulation, on n'observe aucune amélioration sur la fiabilité et la performance du plan binomial. II semble que l'augmentation de l'effort d'échantillonnage doive être plus importante pour pouvoir améliorer les performancess d'un plan binomial pour un seuil élevé.

Le nombre moyen d'échantillons à prélever pour arrêter une décision a été similaire et à peine supérieur au nombre minimal requis pour tous les plans proposés (tableau 3). Sur ce point, les 
plans binomiaux sont aussi efficaces que les plans par décompte. Bien que les avantages de plans d'échantillonnage strictement basés sur la présence ou l'absence de ravageurs soient en partie perdus à cause du décompte nécessaire de 5 thrips plant $^{-1}$, une forte réduction du temps requis pour le décompte est malgré tout encore possible. Dans les situations d'indécision, le nombre maximum de thrips à dénombrer est de 200 et 170 individus pour les seuils de 0,9 et 2,2 thrips feuille ${ }^{-1}$, respectivement. Ceci représente une réduction possible de 62 et $88 \%$ de l'effort de comptage par rapport aux 528 et 1427 thrips requis par les plans par décompte. Ceci est un gain considérable sachant que l'effort de comptage est l'étape la plus longue de l'échantillonnage des thrips.

Dans les situations d'indécision, Sterling et Pieters (1974) proposent d'appliquer la décision de la limite d'acceptation la plus rapprochée au moment du dernier échantillon. Appliqué à nos résultats, le risque d'erreur total pour ces décisions dites terminales est supérieur à $15 \%$ pour tous les plans (tableau 3 ). Comme ce taux est supérieur à un niveau acceptable, un suivi régulier d'un tel site par des échantillonnages rapprochés, tel que préconisé par Sevacherian et Stern (1972), nous apparaît une solution plus en accord avec les principes de la lutte intégrée.

La fiabilité des plans d'échantillonnage séquentiel proposés a été démontrée par leur utilisation en situation réelle. La combinaison de données prélevées dans plusieurs champs aux caractéristiques agronomiques différentes (cultivars, dimensions, niveaux d'infestation du T. tabaci, non-homogénéité de la densité des thrips dans certains champs) semble procurer une certaine robustesse aux plans d'échantillonnage proposés. L'utilisation des plans binomiaux pour les seuils de 2,2 $\pm 0,6$ thrips feuille ${ }^{-1}(\alpha=10 \%)$ et de 0,9 $\pm 0,2$ thrips feuille ${ }^{-1}(\alpha=5 \%)$ apparait donc comme une solution fiable et surtout rapide pour la formulation de recommandations quant à la nécessité de traitements insecticides contre le T. tabaci sur l'oignon. L'emploi de ces plans résultera en une utilisation raisonnée des insecticides, retardant ainsi le développement possible de résistance, tout en assurant au producteur un rendement maximal de sa production par le biais d'un effort d'échantillonnage optimal.

\section{REMERCIEMENTS}

Cette recherche a été réalisée grâce à une aide financière du ministère de l'Agriculture, des Pêcheries et de I'Alimentation du Québec, dans le cadre du programme d'aide à la recherche en agriculture, pêche et alimentation. Les auteurs tiennent également à remercier le Syndicat des producteurs d'oignons du Québec pour leur soutien financier au début du projet, de même que $M$. Luc Brodeur, directeur de Production en Régie Intégrée du Sud de Montréal Enr. pour ses conseils et son soutien technique.

\section{RÉFÉRENCES}

Bates, B.A., M.J. Weiss, R.B. Carlson et D.K. McBride. 1991. Sequential sampling plan for Limothrips denticornis (Thysanoptera: Thripidae) on spring barley. J. Econ. Entomol. 84: 1630-1634.

Belchinski, E.J. et R.L. Stoltz. 1985. Presence-absence sequential decision plans for Tetranychus urticae (Acari: Tetranychidae) in garden-seed beans, Phaseolus vulgaris. J. Econ. Entomol. 78: 1475-1480.

Boivin, G. et L. Brodeur. 1992. Dépistage systématique des ravageurs: impact sur les pratiques phytosanitaires en cultures maraîchères au Québec. Bulletin technique $n^{\circ} 27$, Agriculture Canada, Station de recherche de Saint-Jean-sur-Richelieu, Québec, 16 pp.

Boivin, G. et C. Vincent. 1983. L'échantillonnage séquentiel en phytoprotection: revue de la méthode. Direction générale de la recherche, Agriculture Canada, Contribution $n^{\circ}$ 923, Station de recherche de Saint-Jean-sur-Richelieu, Québec, 28 pp.

Boyce, K.E. et L.A. Miller. 1953. Overwintering habitats of the onion thrips, Thrips tabaci Lind. (Thysanoptera: Thripidae) in southwestern Ontario. Rep. Entomol. Soc. Ont. 84: 82-86.

C.P.V.Q. 1987. Légumes, protection. Conseil des productions végétales du Québec. 112 pp. 
Daniel, W.W. 1978. Applied nonparametric statistics. Houghton Mifflin Co., Boston. $503 \mathrm{pp}$.

Dunn, O.J. 1964. Multiple comparisons using rank sums. Technometrics 6: 241-252.

Edelson, J.V. 1985. A sampling method for estimating absolute numbers of thrips on onions. Southwest. Entomol. 10: 103106.

Edelson, J.V., B. Cartwright et T.A. Royer. 1986. Distribution and impact of Thrips tabaci (Thysanoptera: Thripidae) on onion. J. Econ. Entomol. 79: 502-505.

Edelson, J.V., B. Cartwright et T.A. Royer. 1989. Economics of controlling onion thrips (Thysanoptera: Thripidae) on onions with insecticides in South Texas. J. Econ. Entomol. 82: 561-564.

Fournier, F. 1993. Seuil économique et programme d'échantillonnage séquentiel pour le thrips de l'oignon (Thrips tabaci Lindeman) sur l'oignon. Mémoire de maîtrise. Université McGill, Montréal. 155 pp.

Fowler, G.W. et A.M. Lynch. 1987. Sampling plans in insect pest management based on Wald's sequential probability ratio test. Environ. Entomol. 16: 345-354

Hollingsworth, C.S. et R.E. Berry. 1982. Regression sampling plan for two-spotted spider mite (Acari: Tetranychidae) in Oregon peppermint. J. Econ. Entomol. 75: 497-500.

Iwao, S. 1968. A new regression method for analysing the aggregation pattern of animal populations. Res. Popul. Ecol. 10: $1-20$.

Iwao, S. 1975. A new method of sequential sampling to classify populations relative to a critical density. Res. Popul. Ecol. 16: 281-288.

Karandinos, M.G. 1976. Optimum sample size and comments on some published formulae. Bull. Entomol. Soc. Am. 22: 417 421.

Kendall, D.M. et J.L. Capinera. 1987. Susceptibility of onion growth stages to onion thrips (Thysanoptera: Thripidae) damage and mechanical defoliation. Environ. Entomol. 16: 859-863.
Lewis, T. 1973. Thrips, their biology, ecology and economic importance. Academic Press, New York. 349 pp.

Maiteki, G.A. et R.J. Lamb. 1987. Sequential decision plan for control of pea aphid, Acyrthosiphon pisum (Homoptera: Aphididae), on field peas in Manitoba. J. Econ. Entomol. 80: 605-607.

North, R.C. et A.M. Shelton. 1986. Ecology of Thysanoptera within cabbage fields. Environ. Entomol. 15: 520-526.

Quartey, S.W. 1982. Population dynamics of the onion thrips, Thrips tabaci Lind., on onions. Ph.D. thesis, Michigan State University, East Lansing, Michigan, 198 pp.

Sevacherian, V. et V.M. Stern. 1972. Sequential sampling plans for Lygus bugs in California cotton fields. Environ. Entomol. 1: 704-710.

Shelton, A.M., J.P. Nyrop, R.C. North, C. Petzoldt et R. Foster. 1987. Development and use of a dynamic sequential sampling program for onion thrips, Thrips tabaci (Thysanoptera: Thripidae), on onions. J. Econ. Entomol. 80: 1051-1056.

Southwood, T.R.E. 1978. Ecological methods. Chapman and Hall, London. 524 pp.

Sterling, W.L. et E.P. Pieters. 1974. A sequential sampling package for key cotton arthropods in Texas. Tex. Agric. Exp. Stn Tech. Rep. 74-32, 28 pp.

Suman, C.L. et S.D. Wahi. 1981. Sequential sampling plan for the onion thrips, 7hrips tabaci (Lind.). Entomon 6: 265-269.

Suman, C.L., S.D. Wahi et N.J. Mohan. 1980. Distribution pattern of onion thrips (Thrips tabaci Lind.). Curr. Sci. (Bangalore) 49: 28-29.

Taylor, L.R. 1961. Aggregation, variance and the mean. Nature (Lond.) 189: 732-735.

Wilson, L.T. et P.M. Room 1983. Clumping patterns of fruit and arthropods in cotton, with implications on binomial sampling. Environ. Entomol. 12: 50-54.

Wilson, L.T., C. Pickel, R.C. Mount et: F.G. Zalom. 1983. Presence-absence sequential sampling for cabbage aphid and green peach aphid (Homoptera: Aphididae) on Brussels sprouts. J. Econ. Entomol. 76: 476-479. 